Supporting Information

\title{
Suspect Screening of Hydrocarbon Surfactants in AFFFs and AFFF-Contaminated Groundwater by High Resolution Mass Spectrometry
}

García, Raymmah Aleyda ${ }^{1}$; Chiaia-Hernández, Aurea C ${ }^{2}$; Lara-Martin, Pablo A. ${ }^{3}$; Loos, Martin ${ }^{4}$; Hollender, Juliane ${ }^{5,6}$; Oetjen Karl, ${ }^{7}$ Higgins Chris $^{7}$ and Field, Jennifer ${ }^{8 *}$

${ }^{1}$ Department of Chemistry, Oregon State University, Corvallis, Oregon, 97331, United States

${ }^{2}$ Institute of Geography and Oeschger Center for Climate Change Research, University of Bern, Bern, Switzerland

${ }^{3}$ Department of Physical Chemistry, University of Cadiz, Faculty of Marine and Environmental Sciences, Campus Rio San Pedro, CEI-MAR, Puerto Real, 11510, Cadiz, Spain

${ }^{4}$ Loos computing, Zürich, Switzerland

${ }^{5}$ Eawag, Swiss Federal Institute of Aquatic Science and Technology, 8600 Dübendorf, Switzerland

${ }^{6}$ Institute of Biogeochemistry and Pollutant Dynamics (IBP), ETH Zurich, 8092 Zurich, Switzerland

${ }^{7}$ Department of Civil and Environmental Engineering, Colorado School of Mines, Golden, Colorado,

80401, United States

${ }^{8}$ Department of Environmental and Molecular Toxicology, Oregon State University, Corvallis, OR, United States:Jennifer.Field@oregonstate.edu

*Corresponding author

Number of Pages: 12

Number of Tables: 3

Number of Figures: 4 


\section{MATERIALS AND METHODS}

Chromatography. Separations were performed on the same liquid chromatography-quadrupole time of flight instrumentation as reported by Barzen-Hanson et al., ${ }^{1}$ and Backe et al. 2013, ${ }^{2}$ which consisted of a propylamine guard column placed in line with a silica guard column that retains anions, zwitterions and cations by ion exchange from the $900 \square \mathrm{L}$ sample injected. However, during the initial phase of this study, separations were also performed on a Zorbax diol guard column instead of the propylamine guard column. Nonionic surfactants including alcohol ethoxylates and octylphenol polyethoxylates were detected only on the propylamine guard column but not on the diol guard column. Propylamine columns are used primarily for separating carbohydrates and sugars through hydrophilic interactions. ${ }^{3-5}$ Oxygen-containing nonionic surfactants were not retained by the diol guard column since it retains analytes by anion exchange. ${ }^{2}$ We hypothesize that the non-bonded electrons on the repeating oxygen units are electrostatically attracted to the positive charge on the propylamine Thus, it is essential to use the propylamine guard column to retain the oxygen-containing nonionic surfactants when injecting $900 \square \mathrm{L}$ of an organic extract onto the orthogonal chromatographic system. However, if the more common reverse-phase columns are used, ${ }^{6}$ retention of nonionic surfactants is also achieved.

Data Processing. Parameters chosen for enviMass were dependent on alkyl $\left[\mathrm{CH}_{2} \mathrm{CH}_{2} ; \Delta \mathrm{m} / \mathrm{z} 28\right]$, ethoxylate $\left[\mathrm{CH}_{2} \mathrm{CH}_{2} \mathrm{O} ; \Delta \mathrm{m} / \mathrm{z} 44\right]$, and glucoside $\left[\mathrm{C}_{6} \mathrm{H}_{12} \mathrm{O}_{5} ; \Delta \mathrm{m} / \mathrm{z}\right.$ 162] repeating units. Note that EnviMAss uses nominal masses (e.g., $\mathrm{m} / \mathrm{z} 28,44$, and 162) to search for series. All files were background subtracted. A minimum of three points was needed in order for a series to be considered. Each peak needed to contain at least 10 centroids per peak and peak width could not exceed 120s. Retention time difference between homologues was $120 \mathrm{~s}$ for alkyl repeating units and $10-30$ s for ethoxylate and glucoside repeating units. 
Figure S1. Preliminary attempts to search for homologous series in Kendrick Mass Defect Plots normalized to both a) EO and b) alkyl units in groundwater Site G.

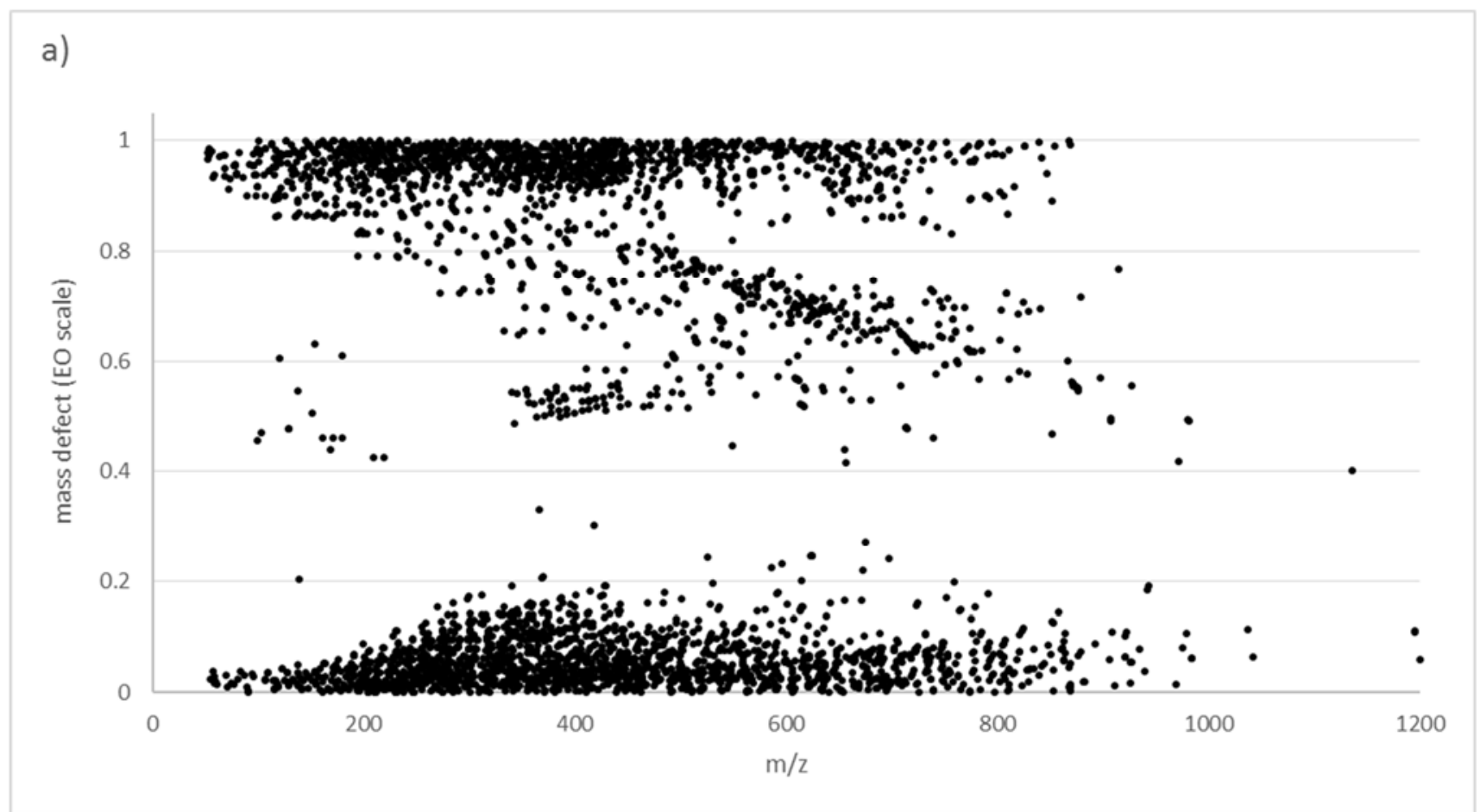

b)

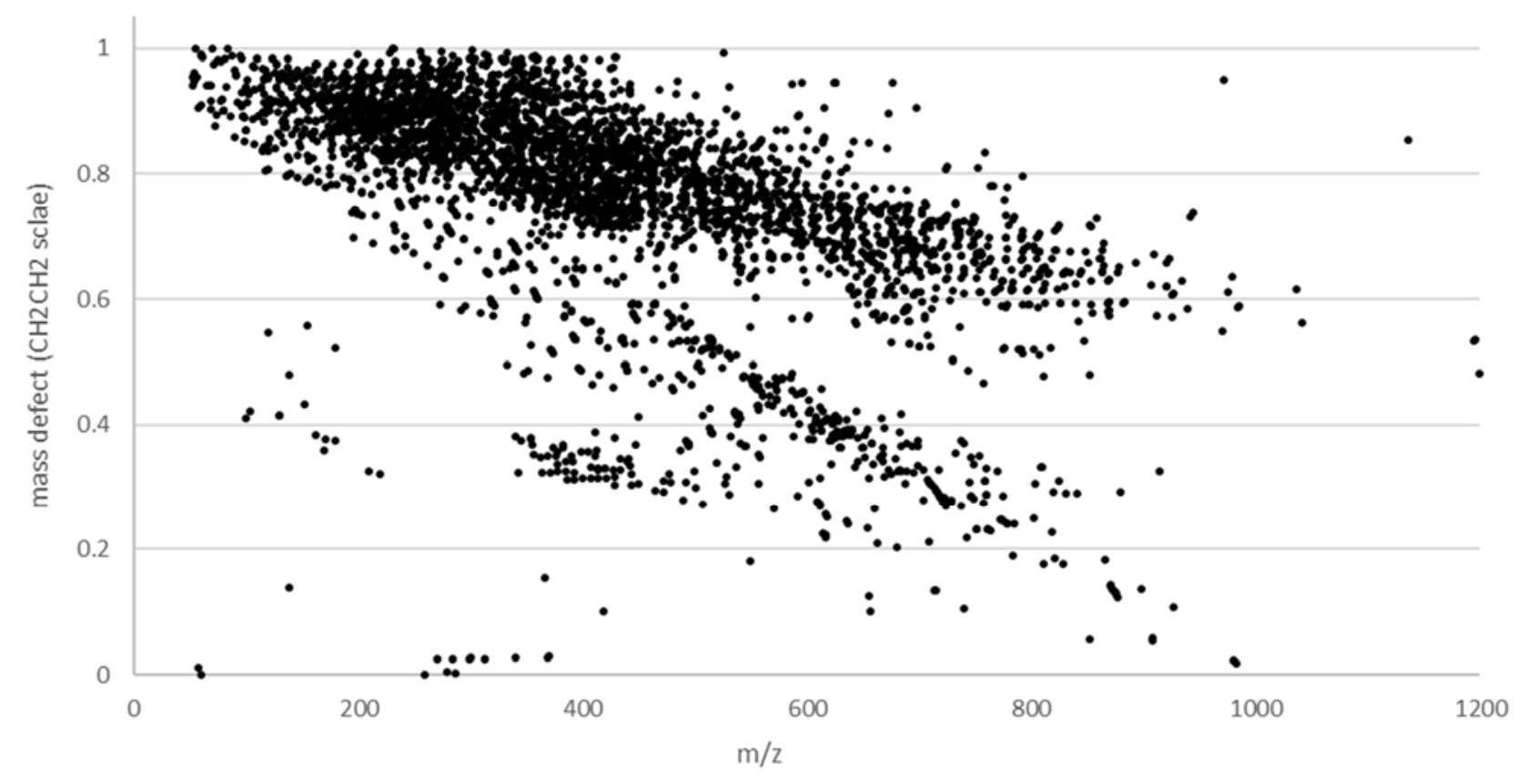


Figure S2. Homologous series of alkyl amine oxides in Reference Material 1 is represented by a nearly vertical line in Figure S1.

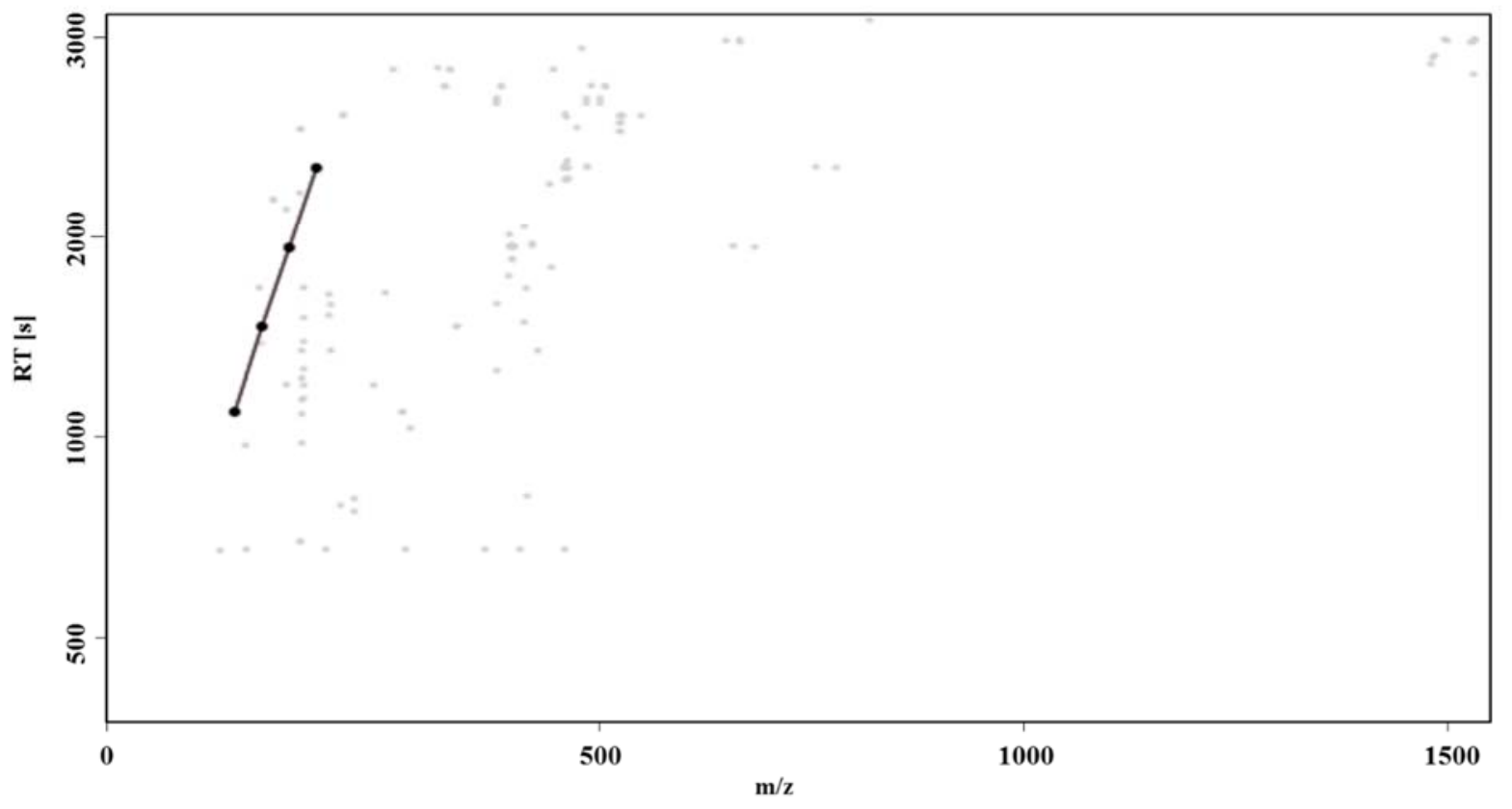


Figure S3. Homologous series of a) repeating alkyl units represented by a nearly vertical line in and b) repeating glucoside units represented by a horizontal line in Reference Material 2, an alkyl polyglucoside. Other homologous series are present in the Reference Material, but where not identified since the main series indicate on the MSDS (dark symbols and line) was successfully identified.
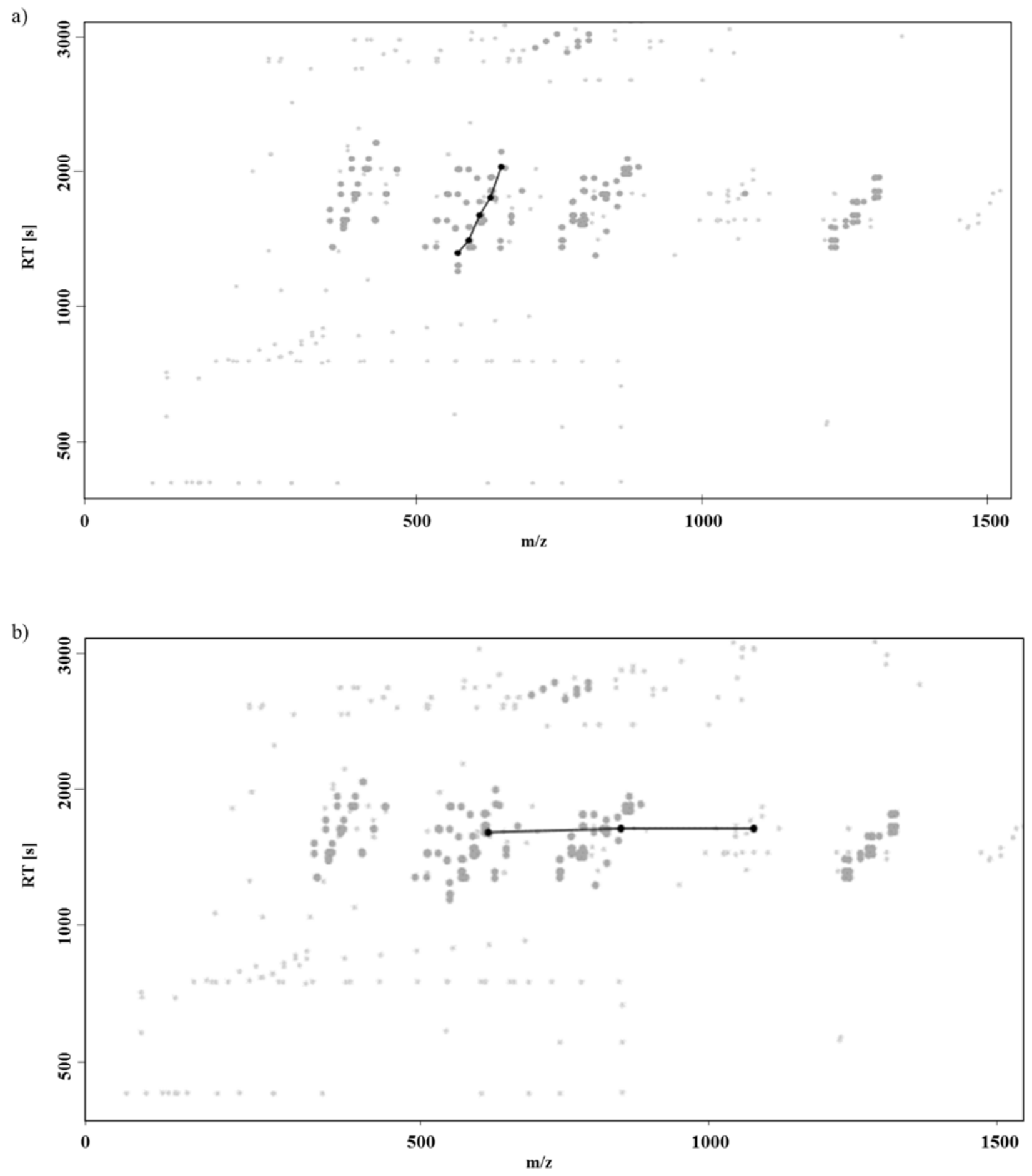
Figure S4. Homologous series in Reference Material 7 (CalFoam ES302) characterized by repeating EO units and a curve. The main homologous series indicated on the MSDS is the series highlighted.

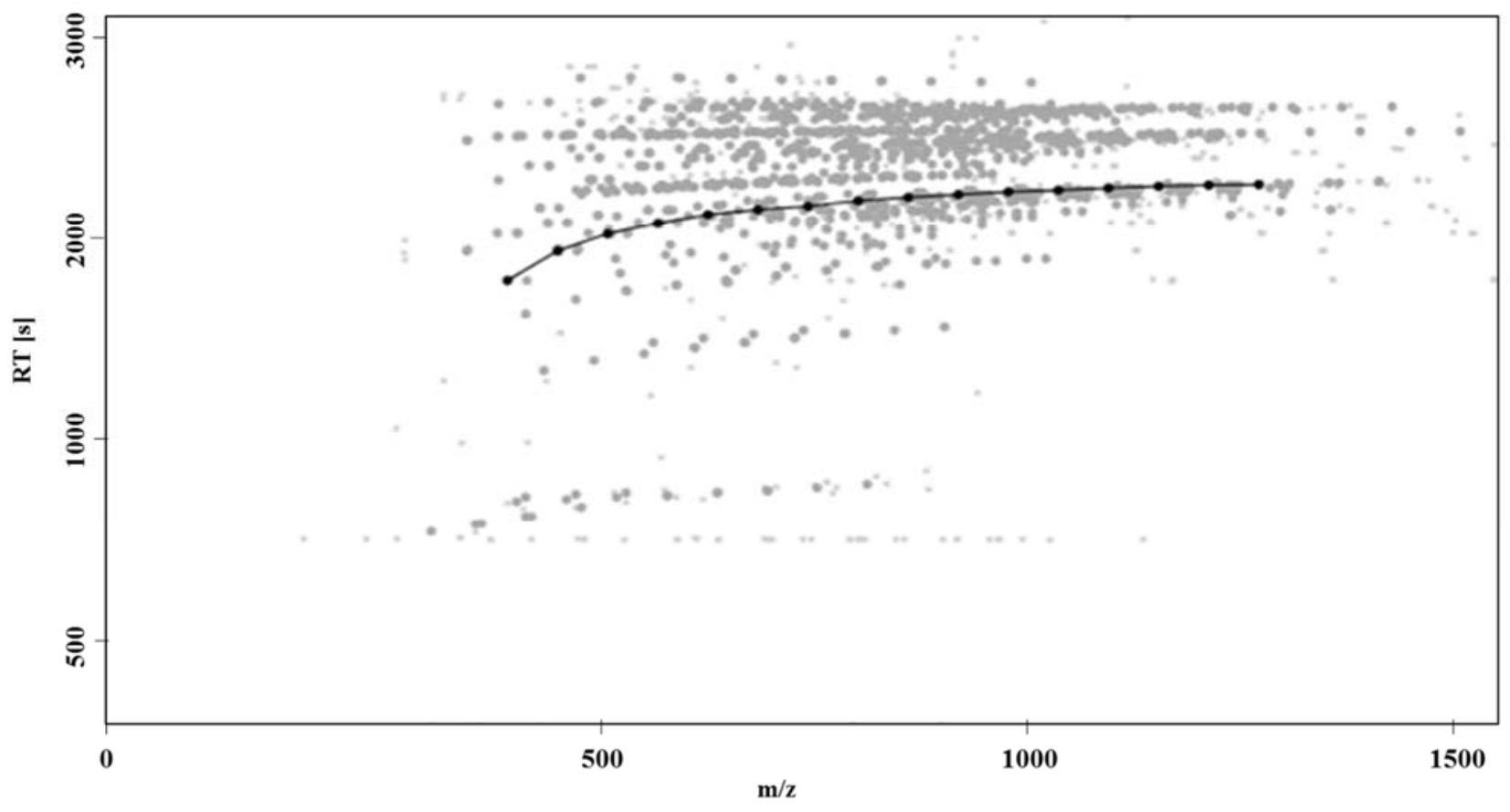


Table S1. Hydrocarbon surfactant classes found in seven reference materials and their respective alkyl (Cn), ethoxylate (EOn), and glucose (GLUCn) repeating units. Multiple repeating units are separated by "/".

\begin{tabular}{|c|c|c|c|c|c|c|c|c|}
\hline $\begin{array}{c}\text { Class } \\
\text { [repeating unit] }\end{array}$ & General Structure & $\begin{array}{c}\text { Reference } \\
\text { Material } 1 \\
\text { (Stepan } \\
\text { Ammonix Lo) }\end{array}$ & $\begin{array}{c}\text { Reference } \\
\text { Material } 2 \\
\text { (BASF APG } 325 \\
\text { N) }\end{array}$ & $\begin{array}{l}\text { Reference } \\
\text { Material } 3 \\
\text { (Cola Teric } \\
\text { CSB) }\end{array}$ & $\begin{array}{c}\text { Reference } \\
\text { Material } 4 \\
\text { (Colonial } \\
\text { TEALS) }\end{array}$ & $\begin{array}{c}\text { Reference } \\
\text { Material } 5 \\
\text { (Lubrizol } \\
\text { Sulfochem } \\
\text { NADS-40) }\end{array}$ & $\begin{array}{c}\text { Reference } \\
\text { Material } 6 \\
\text { (Colonial SOS) }\end{array}$ & $\begin{array}{c}\text { Reference } \\
\text { Material } 7 \\
\text { (CalFoam ES-302) }\end{array}$ \\
\hline $\begin{array}{l}\text { Alkyl amine oxide } \\
\qquad\left[\mathrm{C}_{n}\right]\end{array}$ & $\mathrm{H}-\left.\left.\left[\mathrm{H}_{2} \mathrm{C}\right\}\right|_{\mathrm{n}}\right|_{\mathrm{CH}_{3}} ^{\mathrm{CH}_{3}^{+}-\mathrm{O}_{3}}$ & $10,12,14,16$ & ND & ND & ND & ND & ND & ND \\
\hline $\begin{array}{c}\text { Glucoside } \\
{\left[\mathrm{C}_{\mathrm{n}} / \mathrm{GLUC}_{\mathrm{n}}\right]}\end{array}$ & & ND & $\begin{array}{c}9,10,11 / 1 \\
10 / 1,2,3 \\
11 / 2,3,4\end{array}$ & ND & ND & ND & ND & ND \\
\hline $\begin{array}{c}\text { Cocamidopropyl } \\
\text { hydroxysultaine } \\
{\left[\mathrm{C}_{\mathrm{n}}\right]}\end{array}$ & 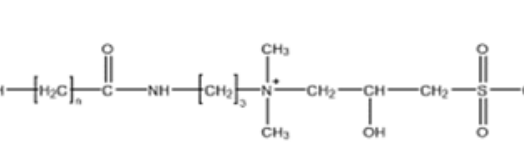 & ND & ND & $7,9,11,13,15$ & ND & ND & ND & ND \\
\hline $\begin{array}{l}\text { Alkyl sulfate } \\
{\left[C_{n}\right]}\end{array}$ & $\left.\mathrm{H}-\mathrm{H}_{2} \mathrm{C}\right\}_{\mathrm{n}} \mathrm{O}-\prod_{\mathrm{S}}^{\mathrm{O}}-\mathrm{O}^{-}$ & ND & ND & ND & $12,14,16$ & $8,9,10,11,12$ & $8,9,10,11$ & ND \\
\hline $\begin{array}{l}\text { Alkyl ether sulfate } \\
\qquad\left[\mathrm{C}_{\mathrm{n}} / \mathrm{EO}_{\mathrm{n}}\right]\end{array}$ & $\left.\left.\mathrm{H}-\mathrm{H}_{2} \mathrm{C}\right\}_{\mathrm{n}} \mathrm{OOC}_{2} \mathrm{H}_{4}\right\}_{\mathrm{n}} \mathrm{O}-\|_{\mathrm{O}}^{\mathrm{S}}-\mathrm{O}^{-}$ & ND & ND & ND & ND & ND & ND & $\mid \begin{array}{c}12 / 1,2,3,4,5,6,7,8 \\
9,10,11\end{array}$ \\
\hline
\end{tabular}


Table S2. Hydrocarbon surfactant classes found in AFFF and groundwater and their respective errors (ppm) and confidence levels.

\begin{tabular}{|c|c|c|c|c|c|c|c|}
\hline Class & $\mathrm{n}$ & $\begin{array}{l}\text { Precursor } \\
\text { neutral } \\
\text { mass }\end{array}$ & Adduct & $\begin{array}{c}\text { observed } \\
\mathrm{m} / \mathrm{z}\end{array}$ & $\begin{array}{l}\text { Error } \\
\text { (ppm) }\end{array}$ & $\begin{array}{c}\text { Confidence } \\
\text { Level } \\
\text { AFFF }\end{array}$ & $\begin{array}{l}\text { Confidence } \\
\text { Level } \\
\text { Groundwater }\end{array}$ \\
\hline \multirow{18}{*}{ 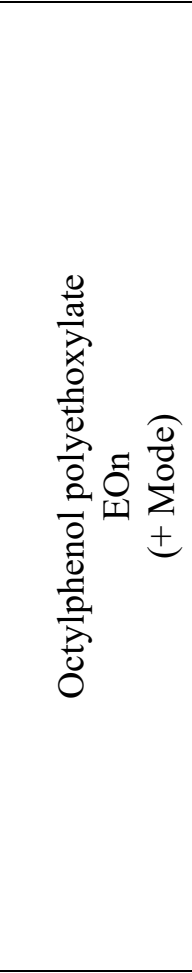 } & 3 & 338.24516 & {$[\mathrm{M}+\mathrm{NH} 4]+$} & 356.2797 & 0.6 & 3 & $2 a$ \\
\hline & 4 & 382.27138 & {$[\mathrm{M}+\mathrm{NH} 4]+$} & 400.3054 & -0.8 & $2 a$ & $2 a$ \\
\hline & 5 & 426.29759 & {$[\mathrm{M}+\mathrm{NH} 4]+$} & 444.332 & 0.1 & $2 b$ & $2 a$ \\
\hline & 6 & 470.32381 & {$[\mathrm{M}+\mathrm{NH} 4]+$} & 488.3556 & -5.3 & $2 a$ & $2 a$ \\
\hline & 7 & 514.35002 & {$[\mathrm{M}+\mathrm{NH} 4]+$} & 532.3837 & -1.4 & $2 a$ & $2 a$ \\
\hline & 8 & 558.37623 & {$[\mathrm{M}+\mathrm{NH} 4]+$} & 576.4096 & -1.7 & $2 a$ & $2 a$ \\
\hline & 9 & 602.40245 & {$[\mathrm{M}+\mathrm{NH} 4]+$} & 620.4357 & -1.8 & $2 a$ & $2 \mathrm{a}$ \\
\hline & 10 & 646.42866 & {$[\mathrm{M}+\mathrm{NH} 4]+$} & 664.4617 & -2.0 & $2 a$ & $2 a$ \\
\hline & 11 & 690.45488 & {$[\mathrm{M}+\mathrm{NH} 4]+$} & 708.4876 & -2.4 & $2 a$ & $2 a$ \\
\hline & 12 & 734.48109 & {$[\mathrm{M}+\mathrm{NH} 4]+$} & 752.5135 & -2.6 & 3 & NA \\
\hline & 13 & 778.50731 & {$[\mathrm{M}+\mathrm{NH} 4]+$} & 796.5399 & -2.2 & 3 & NA \\
\hline & 14 & 822.53352 & {$[\mathrm{M}+\mathrm{NH} 4]+$} & 840.5669 & -1.2 & 3 & NA \\
\hline & 15 & 866.55974 & {$[\mathrm{M}+\mathrm{NH} 4]+$} & 884.5926 & -1.7 & 3 & NA \\
\hline & 16 & 910.58595 & {$[\mathrm{M}+\mathrm{NH} 4]+$} & 928.6182 & -2.3 & 3 & NA \\
\hline & 17 & 954.61217 & {$[\mathrm{M}+\mathrm{NH} 4]+$} & 972.6454 & -1.2 & 3 & NA \\
\hline & 18 & 998.63838 & {$[\mathrm{M}+\mathrm{NH} 4]+$} & 1016.672 & -1.2 & 3 & NA \\
\hline & 19 & 1042.6646 & {$[\mathrm{M}+\mathrm{NH} 4]+$} & 1060.699 & -0.4 & 3 & NA \\
\hline & 20 & 1086.69081 & {$[\mathrm{M}+\mathrm{NH} 4]+$} & 1104.725 & -0.6 & 3 & NA \\
\hline \multirow{9}{*}{ 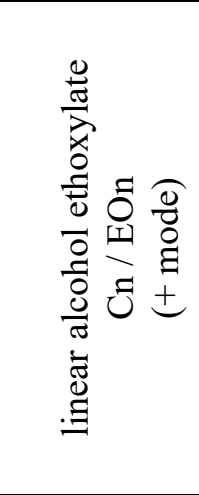 } & $\mathrm{C} 10 \mathrm{EO} 2$ & 246.22016 & {$[\mathrm{M}+\mathrm{NH} 4]+$} & 264.2351 & -1.0 & $2 \mathrm{a}$ & 3 \\
\hline & $\mathrm{C} 10 \mathrm{EO} 3$ & 290.24637 & {$[\mathrm{M}+\mathrm{NH} 4]+$} & 308.2794 & -0.4 & $2 \mathrm{a}$ & 3 \\
\hline & C10 EO4 & 334.27259 & {$[\mathrm{M}+\mathrm{NH} 4]+$} & 352.3052 & -1.5 & $2 \mathrm{a}$ & 3 \\
\hline & C10 EO5 & 378.2988 & {$[\mathrm{M}+\mathrm{NH} 4]+$} & 396.3313 & -1.6 & $2 \mathrm{a}$ & 3 \\
\hline & C10 EO6 & 422.32502 & {$[\mathrm{M}+\mathrm{NH} 4]+$} & 440.3567 & -3.4 & $2 \mathrm{a}$ & 3 \\
\hline & C10 EO7 & 466.35123 & {$[\mathrm{M}+\mathrm{NH} 4]+$} & 484.3829 & -3.1 & $2 \mathrm{a}$ & 3 \\
\hline & C10 EO8 & 510.37745 & {$[\mathrm{M}+\mathrm{NH} 4]+$} & 528.4086 & -3.9 & $2 \mathrm{a}$ & 3 \\
\hline & C10 EO9 & 554.40366 & {$[\mathrm{M}+\mathrm{NH} 4]+$} & 572.4352 & -2.8 & $2 a$ & NA \\
\hline & C10 EO10 & 598.42988 & {$[\mathrm{M}+\mathrm{NH} 4]+$} & 616.4607 & -3.7 & $2 \mathrm{a}$ & NA \\
\hline \multirow{4}{*}{ 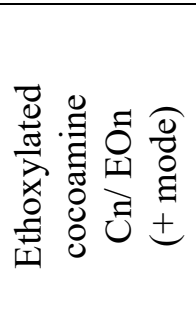 } & 14 & 301.26169 & {$[\mathrm{M}+\mathrm{Na}]+$} & 324.2381 & -0.8 & $2 \mathrm{a}$ & $2 a$ \\
\hline & 15 & 315.26477 & {$[\mathrm{M}+\mathrm{Na}]+$} & 338.2529 & -3.1 & $2 \mathrm{a}$ & $2 a$ \\
\hline & 16 & 329.28042 & {$[\mathrm{M}+\mathrm{Na}]+$} & 352.269 & -2 & $2 \mathrm{a}$ & $2 \mathrm{a}$ \\
\hline & 17 & 343.29607 & {$[\mathrm{M}+\mathrm{Na}]+$} & 366.2854 & -1.6 & $2 a$ & NA \\
\hline
\end{tabular}




\begin{tabular}{|c|c|c|c|c|c|c|c|}
\hline Class & $\mathrm{N}$ & $\begin{array}{l}\text { Precursor } \\
\text { neutral } \\
\text { mass }\end{array}$ & Adduct & $\begin{array}{c}\text { observed } \\
\mathrm{m} / \mathrm{z}\end{array}$ & $\begin{array}{l}\text { Error } \\
(\mathrm{ppm})\end{array}$ & $\begin{array}{l}\text { Confidence } \\
\text { Level AFFF }\end{array}$ & $\begin{array}{c}\text { Confidence } \\
\text { Level } \\
\text { Groundwater }\end{array}$ \\
\hline \multirow{17}{*}{ 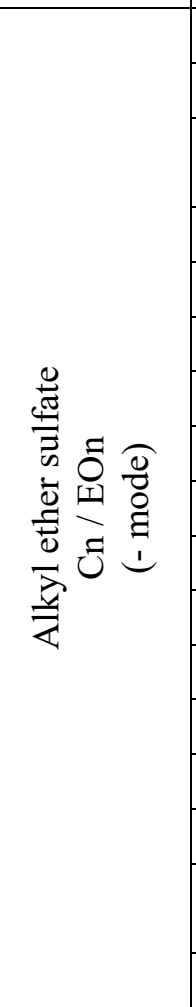 } & C12 EO8 & 618.36504 & [M-H]- & 617.3582 & 0.9 & $2 a$ & NA \\
\hline & C12 EO9 & 662.39124 & [M-H]- & 661.3856 & 2.6 & $2 a$ & NA \\
\hline & $\begin{array}{c}\mathrm{C} 12 \\
\mathrm{EO} 10\end{array}$ & 706.41744 & [M-H]- & 705.4084 & -2.3 & $2 \mathrm{a}$ & NA \\
\hline & C14 EO1 & 338.21284 & [M-H]- & 337.2053 & -0.3 & $2 a$ & NA \\
\hline & $\mathrm{C} 14 \mathrm{EO} 2$ & 382.23904 & [M-H]- & 381.2319 & 0.8 & $2 a$ & NA \\
\hline & C14 EO3 & 426.26524 & [M-H]- & 425.2576 & -0.6 & $2 a$ & NA \\
\hline & C15 EO1 & 352.22844 & [M-H]- & 351.2185 & -7.3 & $2 a$ & $2 a$ \\
\hline & $\mathrm{C} 15 \mathrm{EO} 2$ & 396.25456 & [M-H]- & 395.2446 & -6.8 & NA & $2 a$ \\
\hline & C15 EO3 & 440.31379 & [M-H]- & 439.2732 & -0.6 & NA & $2 a$ \\
\hline & C15 EO4 & 484.30714 & [M-H]- & 483.2998 & 0.2 & NA & $2 a$ \\
\hline & C15 EO5 & 528.33321 & [M-H]- & 527.3257 & -0.4 & NA & $2 a$ \\
\hline & C15 EO6 & 572.35954 & [M-H]- & 571.3522 & 0.0 & NA & $2 a$ \\
\hline & C16 EO1 & 366.23672 & [M-H]- & 365.2371 & 1.0 & $2 \mathrm{a}$ & NA \\
\hline & C16 EO8 & 674.40813 & [M-H]- & 673.4206 & 0.5 & NA & $2 \mathrm{a}$ \\
\hline & C16 EO9 & 718.45384 & [M-H]- & 717.4477 & 1.8 & NA & $2 a$ \\
\hline & $\begin{array}{c}\text { C16 } \\
\text { EO10 }\end{array}$ & 762.47993 & [M-H]- & 761.4727 & 0.0 & NA & $2 a$ \\
\hline & C17EO1 & 380.26019 & [M-H]- & 379.2524 & 0.0 & $2 \mathrm{a}$ & NA \\
\hline \multirow{7}{*}{ 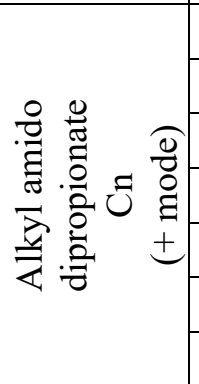 } & 4 & 217.13086 & {$[\mathrm{M}+\mathrm{H}]+$} & 218.1385 & -1.0 & $2 a$ & $2 a$ \\
\hline & 6 & 245.16022 & {$[\mathrm{M}+\mathrm{H}]+$} & 246.1699 & -0.3 & $2 \mathrm{a}$ & $2 \mathrm{a}$ \\
\hline & 8 & 273.19346 & {$[\mathrm{M}+\mathrm{H}]+$} & 275.2083 & -2.9 & $2 a$ & $2 a$ \\
\hline & 10 & 301.22476 & {$[\mathrm{M}+\mathrm{H}]+$} & 303.2405 & 0.4 & $2 a$ & $2 \mathrm{a}$ \\
\hline & 12 & 329.25606 & {$[\mathrm{M}+\mathrm{H}]+$} & 330.2642 & 1.0 & $2 a$ & $2 \mathrm{a}$ \\
\hline & 13 & 343.27171 & {$[\mathrm{M}+\mathrm{H}]+$} & 344.2793 & -0.6 & $2 \mathrm{a}$ & NA \\
\hline & 14 & 357.28736 & {$[\mathrm{M}+\mathrm{H}]+$} & 358.2951 & -0.2 & $2 a$ & NA \\
\hline
\end{tabular}




\begin{tabular}{|c|c|c|c|c|c|c|c|}
\hline Class & $\mathrm{N}$ & $\begin{array}{c}\text { Precursor } \\
\text { neutral mass }\end{array}$ & Adduct & $\begin{array}{c}\text { observed } \\
\mathrm{m} / \mathrm{z}\end{array}$ & $\begin{array}{l}\text { Error } \\
(\mathrm{ppm})\end{array}$ & $\begin{array}{l}\text { Confidence } \\
\text { Level AFFF }\end{array}$ & $\begin{array}{l}\text { Confidence } \\
\text { Level } \\
\text { Groundwater }\end{array}$ \\
\hline \multirow{5}{*}{ 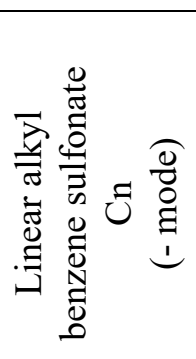 } & 10 & 298.16081 & [M-H]- & 297.1528 & -0.5 & $2 a$ & $2 a$ \\
\hline & 11 & 312.17646 & [M-H]- & 311.1687 & 0.2 & $2 a$ & $2 a$ \\
\hline & 12 & 326.19211 & [M-H]- & 325.1846 & 0.9 & $2 a$ & $2 a$ \\
\hline & 13 & 340.20776 & {$[\mathrm{M}-\mathrm{H}]-$} & 339.1999 & 0.0 & $2 a$ & $2 a$ \\
\hline & 14 & 354.22341 & [M-H]- & 353.2177 & 6.0 & NA & $2 a$ \\
\hline \multirow{4}{*}{ 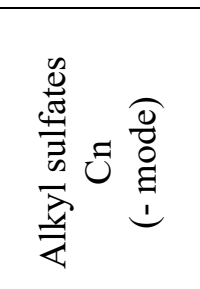 } & 8 & 210.09313 & [M-H]- & 209.0854 & 0.6 & 1 & NA \\
\hline & 9 & 224.10878 & [M-H]- & 223.1009 & -0.2 & 1 & NA \\
\hline & 10 & 238.12443 & [M-H]- & 237.1166 & -0.1 & 1 & NA \\
\hline & 12 & 266.15573 & [M-H]- & 265.1479 & -0.1 & 1 & NA \\
\hline \multirow{6}{*}{ 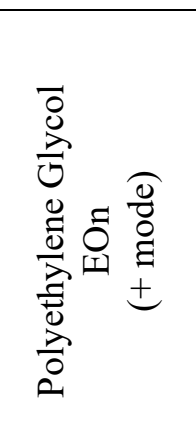 } & 8 & 370.21973 & {$[\mathrm{M}+\mathrm{NH} 4]+$} & 388.2542 & 0.1 & $2 a$ & NA \\
\hline & 9 & 414.24595 & {$[\mathrm{M}+\mathrm{NH} 4]+$} & 432.2801 & -0.5 & $2 a$ & NA \\
\hline & 10 & 458.27216 & {$[\mathrm{M}+\mathrm{NH} 4]+$} & 476.3063 & -0.6 & $2 a$ & NA \\
\hline & 11 & 502.29838 & {$[\mathrm{M}+\mathrm{NH} 4]+$} & 520.3333 & 1.0 & $2 a$ & NA \\
\hline & 12 & 546.32459 & {$[\mathrm{M}+\mathrm{NH} 4]+$} & 564.3595 & 1.0 & $2 \mathrm{a}$ & NA \\
\hline & 13 & 590.35081 & {$[\mathrm{M}+\mathrm{NH} 4]+$} & 608.3857 & 0.8 & $2 a$ & NA \\
\hline \multirow{4}{*}{ 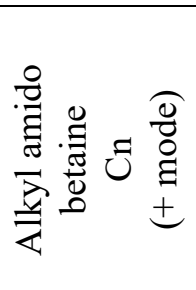 } & 4 & 245.1860 & {$[\mathrm{M}+\mathrm{H}]+$} & 246.1938 & -0.1 & 3 & $2 a$ \\
\hline & 5 & 259.20162 & {$[\mathrm{M}+\mathrm{H}]+$} & 260.2077 & -6.6 & 3 & $2 a$ \\
\hline & 6 & 273.21727 & {$[\mathrm{M}+\mathrm{H}]+$} & 274.2273 & 7.9 & 3 & $2 a$ \\
\hline & 7 & 287.23292 & {$[\mathrm{M}+\mathrm{H}]+$} & 288.2404 & -1.2 & $2 b$ & $2 \mathrm{a}$ \\
\hline \multirow{4}{*}{ 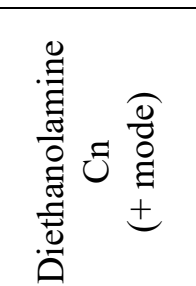 } & 7 & 231.18344 & {$[\mathrm{M}+\mathrm{H}]+$} & 232.1902 & -2.1 & NA & $2 a$ \\
\hline & 9 & 259.213531 & {$[\mathrm{M}+\mathrm{H}]+$} & 260.222 & 1.9 & NA & $2 a$ \\
\hline & 11 & 287.24604 & {$[\mathrm{M}+\mathrm{H}]+$} & 288.2531 & -0.7 & NA & $2 a$ \\
\hline & 13 & 315.27734 & {$[\mathrm{M}+\mathrm{H}]+$} & 316.2844 & -0.8 & NA & $2 \mathrm{a}$ \\
\hline
\end{tabular}

$\mathrm{NA}=$ Not available indicating the homologous series was not present in either AFFF or groundwater. 
Table S3. Summary of surfactants detected in AFFFs and AFFF-impacted groundwater.

\begin{tabular}{|c|c|c|c|}
\hline Surfactant & Structure & $\begin{array}{c}\text { Archived } \\
\text { AFFFs }\end{array}$ & Groundwaters \\
\hline $\begin{array}{c}\text { Octylphenol } \\
\text { polyethoxylates }\end{array}$ & $\begin{array}{c}\text { Linear alcohol } \\
\text { ethoxylates }\end{array}$ \\
\hline $\begin{array}{c}\text { Ethoxylated } \\
\text { cocoamines }\end{array}$ & & $\checkmark$ & $\checkmark$ \\
\hline $\begin{array}{c}\text { Alkyl ether } \\
\text { sulfates }\end{array}$ \\
\hline $\begin{array}{c}\text { Alkyl amido } \\
\text { dipropionates }\end{array}$
\end{tabular}




\section{References}

1. Barzen-Hanson, K. A.; Roberts, S. C.; Choyke, S.; Oetjen, K.; McAlees, A.; Riddell, N.; McCrindle, R.; Ferguson, P. L.; Higgins, C. P.; Field, J. A., Discovery of 40 classes of per- and polyfluoroalkyl substances in historical aqueous film-forming foams (AFFF) and AFFF-impacted groundwater. Environ Sci Technol 2017, 51, (4), 2047-2057.

2. Backe, W. J.; Day, T. C.; Field, J. A., Zwitterionic, cationic, and anionic fluorinated chemicals in aqueous film forming foam formulations and groundwater from us military bases by nonaqueous large-volume injection HPLC-MS/MS. Environmental Science and Technology 2013, 47, (10), 52265234.

3. Jandera, P., Stationary and mobile phases in hydrophilic interaction chromatography: a review. Analytica Chimica Acta 2011, 692, (1-2), 1-25.

4. Hao, Z. G.; Xiao, B. M.; Weng, N. D., Impact of column temperature and mobile phase components on selectivity of hydrophilic interaction chromatography (HILIC). J. Sep. Sci. 2008, 31, (9), 14491464.

5. Agilent Technologies, Column Selection Guide for HPLC. In 2012.

6. Ferrer, I.; Thurman, E. M., Analysis of hydraulic fracturing additives by LC/Q-TOF-MS. Anal Bioanal Chem 2015, 407, (21), 6417-28. 